\title{
Meta-analysis of STAT4 and IFIH1 polymorphisms in type 1 diabetes mellitus patients with autoimmune polyglandular syndrome type III
}

\author{
J. de Azevêdo Silva', N.A.C. Tavares ${ }^{1}$, M.M.S. Santos ${ }^{1,2}$, R. Moura ${ }^{1,2}$, \\ R.L. Guimarães ${ }^{1,2}$, J. Araújo ${ }^{3}$, S. Crovella ${ }^{1,2}$ and L.A.C. Brandão ${ }^{2,4}$ \\ 'Laboratório de Imunopatologia Keizo Asami, Universidade Federal de Pernambuco, \\ Recife, PE, Brasil \\ 'Departamento de Genética, Universidade Federal de Pernambuco, \\ Recife, PE, Brasil \\ ${ }^{3}$ Hospital das Clínicas, Unidade de Endocrinologica Pediátrica, \\ Universidade Federal de Pernambuco, Recife, PE, Brasil \\ ${ }^{4}$ Departamento de Patologia, Universidade Federal de Pernambuco, \\ Recife, PE, Brasil \\ Corresponding author: J. de Azevêdo Silva \\ E-mail: j.azvedo@gmail.com
}

Genet. Mol. Res. 14 (4): 17730-17738 (2015)

Received August 3, 2015

Accepted September 30, 2015

Published December 21, 2015

DOI http://dx.doi.org/10.4238/2015.December.21.46

ABSTRACT. Type 1 diabetes mellitus (T1D) is an organ-specific autoimmune disease characterized by T-cell mediated self-destruction of insulinproducing $\beta$ cells in the pancreas. T1D patients are prone to develop other glandular autoimmune disorders, such as autoimmune thyroid disease that occurs simultaneously with autoimmune polyglandular syndrome type III (APSIII). Signal transducer and activator of transcription 4 (STAT4) is a wellknown regulator of proinflammatory cytokines, and interferon-induced with helicase $\mathrm{C}$ domain 1 (IFIH1) is activated in the interferon type I response. Both genes have been examined separately in autoimmune diseases and, 
in this study, we assessed their joint role in T1D and APSIII. We conducted a case-control study, enrolling 173 T1D patients and 191 healthy controls from northeastern Brazil, to assess the distribution of the rs7574865 and rs3024839 SNPs in STAT4 and the rs3747517 and rs1990760 SNPs in IFIH1 in T1D and APSIII patients. Additionally, we conducted a metaanalysis with the rs7574865 SNP in STAT4 (1392 T1D patients and 1629 controls) and the rs1990760 SNP in IFIH1 (25092 T1D patients and 28544 controls) to examine their association with T1D. Distribution of STAT4 and IFIH1 allelic frequencies did not show statistically significant differences between T1D patients and controls in our study population; however, the meta-analysis indicated that SNPs in STAT4 and IFIH1 are associated with T1D worldwide. Our findings indicate that although STAT4 and IFIH1 SNPS are not associated with T1D in a Brazilian population, they might play a role in susceptibility to T1D on a larger worldwide scale.

Key words: STAT4; IFIH1; Autoimmune polyglandular syndrome type III; SNP; Type 1 diabetes

\section{INTRODUCTION}

Type 1 diabetes mellitus (T1D) is an organ-specific autoimmune disease characterized by T cell-mediated attack of the insulin-producing $\beta$ cells in the pancreas, leading to insulin deficiency (Gillespie, 2006). T1D is a multifactorial disease caused by genetic and environmental factors, as well as their interaction, that play a key role in the development of the disease. In up to one-quarter of T1D patients, an unbalanced immune system leads to autoimmune polyglandular syndrome type III (APSIII), which is characterized by the simultaneous occurrence of autoimmune thyroid disease (AITD) and sex bias (adult females are preferentially affected) (Kordonouri et al., 2002; Dittmar and Kahaly, 2010). Furthermore, patients with APSIII may also be diagnosed with celiac disease (CD). Despite the genetic variation in human leukocyte antigen (HLA) and its involvement in T1D development, new genes have been identified as potentially important in disease's susceptibility and modulation outside HLA range (Gillespie, 2006; Liang et al., 2012).

Signal transducer and activator of transcription 4 (STAT4) is a latent cytoplasmic transcription factor activated by phosphorylation in response to proinflammatory cytokines, such as interleukin (IL)-12, IL-15, and IL-23 (Levy and Darnell, 2002). STAT4 is involved in T helper 1 (Th1) cell regulation and is expressed in activated peripheral blood monocytes, dendritic cells, and macrophages at inflammation sites. Additionally, STAT4 mediates IL-12 signaling, which modulates Th1 cell differentiation and proliferation, interferon- $\gamma$ (INF- $\gamma$ ) production, and development of T helper 17 (Th17) cells (Kobayashi et al., 2008; Zervou et al., 2008). Since Th1 cells are critical effectors of chronic inflammation disorders, STAT4 could play a pivotal role in the pathogenesis of immune diseases (Kobayashi et al., 2008; Zervou et al., 2008; Bi et al., 2013; Zheng et al., 2013). In fact, single nucleotide polymorphisms (SNPs) within STAT4 (chromosome location: 2q32.2-q32.3) have been reported to be associated with increased risk for several autoimmune diseases (Liang et al., 2012), including rheumatoid arthritis (RA) (Stark et al., 2009), systemic lupus erythematosus (SLE) (Kobayashi et al., 2008), and Sjögren's syndrome (SS) (Palomino-Morales et al., 2010). 
Viral infections have been implicated as triggers in autoimmune disorders in T1D (Jun and Yoon, 2001; Salminen et al., 2003). Interferon-induced with helicase C domain 1 (IFIH1; gene located at chromosome 2q24), also known as MDA5, activates the type I interferon (IFN-I) pathway and pro-inflammatory cytokines by its CARD domains after detecting double-stranded RNA viruses (Chistiakov, 2010). Enterovirus infections, particularly coxsackievirus B4 strains, are known to be T1D-associated; therefore, IFIH1 may play an important role in the development of T1D and its autoimmune-related disorders (Jaïdane et al., 2009). Interestingly, during IFN-I activation, IFIH1 and STAT4 share a common pathway and, since both genes are known to be associated with T1D (Zheng et al., 2013), one can hypothesize that defects in both genes may increase susceptibility to disease compared to defects in just one gene, resulting in a cumulative effect of genetic mutations.

In this study, we investigated the single nucleotide polymorphisms (SNPs) rs7574865 ( $\mathrm{G}>\mathrm{T}$ ) and rs3024839 (T > C) in STAT4 and rs3747517 (C > T) and rs1990760 (C > T) in IFIH1 and their link to T1D and APSIII susceptibility in a northeast Brazilian population. Additionally, we performed a meta-analysis for the rs7574865 and rs1990760 SNPs in T1D predisposition.

\section{MATERIAL AND METHODS}

\section{Patients and control subjects}

We performed a case-control study in T1D patients from Pernambuco State in northeast Brazil. We enrolled 173 T1D patients ranging in age from 0 to 18 years at diagnosis, with an age of $7.3 \pm 4.1$ (means $\pm S D$ ) years at disease onset. The patients attended one of three pediatric endocrinology departments in the public healthcare system in Recife, Brazil (Instituto de Medicina Integral Professor Fernando Figueira, Hospital da Restauração and Hospital das Clínicas). A consent form was obtained from all patients (or their legal representative) enrolled in this study. T1D patients were diagnosed according to American Diabetes Association (ADA) criteria and classified as T1D from clinical and pathological presentation (Gabir et al., 2000).

From the T1D patient group, $47(27.2 \%)$ were diagnosed with APSIII. AITD was diagnosed using antibodies against thyroperoxidase (anti-TPO) and detection was performed using chemiluminescence (Immulite anti-TPO, Diagnostic Products Co., Los Angeles, CA, USA) following the manufacturer instructions. The individuals positive for TPO (titer exceeding 35 $\mathrm{IU} / \mathrm{mL}$, according to indications provided by the manufacturer) were considered as presenting AITD. The control group consisted of 191 healthy unrelated blood donors from the same geographic region with no history of autoimmune or chronic disease. The age of the control group ranged from 16 to 72 years and the means \pm SD age was $38.8 \pm 14.7$ years. This study was carried out with advanced approval from the local Ethics Committee (IMIP Nos. 762/2006 and 1717/2010).

\section{Genotyping}

Genomic DNA was obtained from whole blood and the extraction protocol was performed according to the manufacturer instructions (Wizard Genomic DNA Purification Kit; Promega, Madison, MA, USA). The DNA was stored at $-20^{\circ} \mathrm{C}$ until analysis. The SNPs assessed in this study 
have frequently been described in the literature: rs7574865 (G > T) from intron 3, rs3024839 ( $T$ > C) from exon 4 in STAT4, rs3747517 (C > T) in exon 13, and rs1990760 (C > T) in exon 15 of IFIH1. Genotyping was performed using commercially available Taqman probes and the ABI7500 realtime PCR system (Applied Biosystems, Foster City, CA, USA). The allelic discrimination protocol was performed as recommended by the manufacturer and analyzed using SDS 2.3 software (Applied Biosystems).

\section{Statistics and meta-analysis}

Association analyses were performed by the chi-squared $\left(\chi^{2}\right)$ test with continuity correction, and the odds ratio (OR) and $95 \%$ confidence intervals $(\mathrm{Cl})$ were calculated using the Fisher test. For the meta-analysis, we searched peer-reviewed articles published between 2003 and 2015 (last search performed in July 2015) using logical equations with the following key words on PubMed and Web of Knowledge: "[(IFIHI or MDA5) and T1D]" or "(STAT4 and T1D)". We selected only case-control studies with the allele counts available for both SNPs analyzed (rs7574865 and rs1990760). The meta-analysis tests were carried out using the "Metafor" package (Viechtbauer, 2010). When the $P$ value from the Cochran $Q$ test for heterogeneity was lower than 0.1 , the DerSimonian-Laird's estimator was used for the random-effect or fixedeffect models when necessary. The Haploview version 4.2 software was used for calculation of haplotype associations. Power analyses were performed using the $G^{*}$ Power 3.1.3 software (http://www.psycho.uniduesseldorf.de).

\section{RESULTS}

The allele and genotype frequencies of the SNPs in STAT4 and IFIH1 in patients (T1D + AITD + CD, T1D only, and APSIII) and healthy controls are shown in Table 1. All polymorphisms were in Hardy-Weinberg equilibrium in all groups except the T1D only group. Distribution of STAT4 and IFIH1 genotype and allele frequencies did not show statistically significant differences between patients and controls, indicating no association of the SNPs with development of T1D or APSIII regardless of the genetic model used. These results are shown in Table 2. Of note, the examined SNPs did not show linkage disequilibrium.

Furthermore, we performed a meta-analysis for the rs 7574865 and rs 1990760 SNPs from STAT4 and IFIH1, respectively. Including the present study, we gathered seven publications with data on the rs7574865 SNP (Lee et al., 2008; Martínez et al., 2008a; Zervou et al., 2008; Howson et al., 2011; Park et al., 2011; Bi et al., 2013) and ten for the rs1990760 SNP (Smyth et al., 2006, 2008; Martínez et al., 2008b; Aminkeng et al., 2009; Jermendy et al., 2009; Liu et al., 2009; Schulte et al., 2010; Yang et al., 2012; Bouças et al., 2013; Zurawek et al., 2015). The total number of cases and controls was 1392 and 1629 for the rs7574865 SNP and 25,092 and 28,544 for the rs 1990760 SNP, respectively.

The forest plots of the meta-analyses of the rs7574865 and rs 1990760 SNPs are shown in Figures 1 and 2, respectively. The rs7574865 $(\mathrm{OR}=1.37 ; 95 \% \mathrm{Cl}=1.23-1.52 ; \mathrm{P}<0.0001)$ and $\mathrm{rs} 1990760(\mathrm{OR}=0.85 ; 95 \% \mathrm{Cl}=0.81-0.89 ; \mathrm{P}<0.0001) \mathrm{SNPs}$ were both associated with T1D, although some moderate heterogeneity was detected $\left(I^{2}=43.35 \% ; P_{|Q|}=0.0478\right)$ for the rs1990760 SNP. 
Table 1. Allele and genotype frequencies of the STAT4 (rs7574865 and rs3024839) and IFIH1 (rs3747517 and rs1990760) SNPs in type 1 diabetes mellitus patients (T1D only), T1D patients with autoimmune thyroid disease (AITD) and celiac disease (CD), autoimmune polyglandular syndrome type III (APSIII) patients, and healthy controls (HC).

\begin{tabular}{|c|c|c|c|c|c|c|c|c|}
\hline \multirow[t]{2}{*}{ SNP } & \multicolumn{2}{|c|}{$\mathrm{HC}$} & \multicolumn{2}{|c|}{ T1D + AITD + CD } & \multicolumn{2}{|c|}{ T1D only } & \multicolumn{2}{|c|}{ APSIII } \\
\hline & $N$ & Freq. & $\mathrm{N}$ & Freq. & $\mathrm{N}$ & Freq. & $\mathrm{N}$ & Freq. \\
\hline \multicolumn{9}{|c|}{ rs7574865 } \\
\hline G & 294 & 0.77 & 260 & 0.75 & 197 & 0.78 & 63 & 0.68 \\
\hline $\mathrm{T}$ & 88 & 0.23 & 86 & 0.25 & 57 & 0.22 & 29 & 0.32 \\
\hline GG & 112 & 0.59 & 98 & 0.57 & 76 & 0.60 & 22 & 0.48 \\
\hline GT & 70 & 0.37 & 64 & 0.37 & 45 & 0.35 & 19 & 0.41 \\
\hline TT & 9 & 0.05 & 11 & 0.06 & 6 & 0.05 & 5 & 0.11 \\
\hline \multicolumn{9}{|c|}{ rs3024839 } \\
\hline T & 353 & 1.00 & 331 & 1.00 & 245 & 1.00 & 86 & 1.00 \\
\hline C & 1 & 0.00 & 1 & 0.00 & 1 & 0.00 & 0 & 0.00 \\
\hline TT & 176 & 0.99 & 165 & 0.99 & 122 & 0.99 & 43 & 1.00 \\
\hline TC & 1 & 0.01 & 1 & 0.01 & 1 & 0.01 & 0 & 0.00 \\
\hline $\mathrm{CC}$ & 0 & 0.00 & 0 & 0.00 & 0 & 0.00 & 0 & 0.00 \\
\hline \multicolumn{9}{|c|}{ rs3747517 } \\
\hline C & 230 & 0.68 & 249 & 0.72 & 179 & 0.72 & 70 & 0.74 \\
\hline $\mathrm{T}$ & 110 & 0.32 & 95 & 0.28 & 71 & 0.28 & 24 & 0.26 \\
\hline $\mathrm{CC}$ & 77 & 0.45 & 87 & 0.51 & 63 & 0.50 & 24 & 0.51 \\
\hline CT & 76 & 0.45 & 75 & 0.44 & 53 & 0.42 & 22 & 0.47 \\
\hline TT & 17 & 0.10 & 10 & 0.06 & 9 & 0.07 & 1 & 0.02 \\
\hline \multicolumn{9}{|c|}{ rs1990760 } \\
\hline C & 202 & 0.61 & 201 & 0.60 & 150 & 0.61 & 51 & 0.57 \\
\hline $\mathrm{T}$ & 130 & 0.39 & 135 & 0.40 & 96 & 0.39 & 39 & 0.43 \\
\hline CC & 59 & 0.36 & 67 & 0.40 & 52 & 0.42 & 15 & 0.33 \\
\hline CT & 84 & 0.51 & 67 & 0.40 & 46 & 0.37 & 21 & 0.47 \\
\hline TT & 23 & 0.14 & 34 & 0.20 & 25 & 0.20 & 9 & 0.20 \\
\hline
\end{tabular}

Table 2. Odds ratios, $95 \%$ confidence intervals, and $P$ values from the association analysis between the STAT4 (rs7574865 and rs3024839) and IFIH1 (rs3024839 and rs1990760) variants and type 1 diabetes (T1D) and autoimmune polyglandular syndrome type III (APSIII).

\begin{tabular}{|c|c|c|c|c|c|c|c|c|c|c|}
\hline \multirow[t]{2}{*}{ Comparison } & \multicolumn{3}{|c|}{$11 \times 01 \times 00$} & \multicolumn{3}{|c|}{$01 \times 00$} & \multicolumn{3}{|c|}{$11 \times 00$} & \multirow{2}{*}{$\begin{array}{l}1 \times 0 \\
P \text { value }\end{array}$} \\
\hline & $P$ value & OR & $95 \% \mathrm{Cl}$ & $P$ value & OR & $95 \% \mathrm{Cl}$ & $P$ value & OR & $95 \% \mathrm{Cl}$ & \\
\hline \multicolumn{11}{|l|}{ rs7574865 } \\
\hline $\mathrm{HC}$ vs T1D & 0.7737 & 1.04 & $(0.68-1.61)$ & 0.9302 & 1.40 & $(0.56-3.51)$ & 0.6320 & 1.11 & $(0.79-1.55)$ & 0.6258 \\
\hline $\mathrm{HC}$ vs T1D only & 0.9753 & 0.95 & $(0.59-1.52)$ & 0.9184 & 0.98 & $(0.34-2.87)$ & 0.8095 & 0.97 & $(0.66-1.41)$ & 0.9371 \\
\hline HC vs APSIII & 0.1864 & 1.38 & $(0.7-2.73)$ & 0.4507 & 2.83 & $(0.86-9.25)$ & 0.1570 & 1.54 & $(0.93-2.54)$ & 0.1188 \\
\hline T1D only vs APSIII & 0.2038 & 1.46 & $(0.71-2.98)$ & 0.3947 & 2.88 & $(0.8-10.34)$ & 0.1910 & 1.59 & $(0.94-2.7)$ & 0.1127 \\
\hline \multicolumn{11}{|l|}{ rs3024839 } \\
\hline $\mathrm{HC}$ vs T1D & ND & 1.07 & $(0.09-23.29)$ & 0.5067 & ND & ND & ND & 1.07 & $(0.07-17.12)$ & 0.5073 \\
\hline $\mathrm{HC}$ vs T1D only & ND & 1.44 & $(0.09-23.29)$ & 0.6444 & ND & ND & ND & 1.44 & $(0.09-23.15)$ & 0.6449 \\
\hline HC vs APSIII & ND & 0.00 & ND & 0.4415 & ND & ND & ND & 0.00 & ND & 0.4420 \\
\hline T1D only vs APSIII & ND & 0.00 & ND & 0.5812 & ND & ND & ND & 0.00 & ND & 0.5817 \\
\hline \multicolumn{11}{|l|}{ rs3747517 } \\
\hline HC vs T1D & 0.2983 & 0.87 & $(0.53-1.38)$ & 0.6264 & 0.52 & $(0.22-1.2)$ & 0.1821 & 0.80 & $(0.57-1.11)$ & 0.2046 \\
\hline $\mathrm{HC}$ vs T1D only & 0.5700 & 0.85 & (0.53-1.38) & 0.6000 & 0.65 & $(0.27-1.55)$ & 0.4438 & 0.83 & $(0.58-1.18)$ & 0.3480 \\
\hline HC vs APSIII & 0.2176 & 0.93 & $(0.48-1.8)$ & 0.9589 & 0.19 & $(0.02-1.49)$ & 0.1519 & 0.72 & $(0.43-1.2)$ & 0.2539 \\
\hline T1D only vs APSIII & 0.4346 & 1.09 & $(0.55-2.16)$ & 0.9433 & 0.29 & $(0.04-2.43)$ & 0.4108 & 0.86 & $(0.5-1.48)$ & 0.6929 \\
\hline \multicolumn{11}{|l|}{ rs1990760 } \\
\hline $\mathrm{HC}$ vs T1D & 0.1037 & 0.70 & $(0.37-1.04)$ & 0.1805 & 1.30 & $(0.69-2.45)$ & 0.5124 & 1.04 & $(0.77-1.42)$ & 0.8486 \\
\hline $\mathrm{HC}$ vs T1D only & 0.0688 & 0.62 & $(0.37-1.04)$ & 0.0941 & 1.23 & $(0.63-2.43)$ & 0.6645 & 0.99 & $(0.71-1.39)$ & 0.9569 \\
\hline HC vs APSIII & 0.5943 & 0.98 & $(0.47-2.06)$ & 0.8848 & 1.54 & $(0.59-4.01)$ & 0.5259 & 1.19 & $(0.74-1.9)$ & 0.5512 \\
\hline T1D only vs APSIII & 0.5036 & 1.58 & $(0.73-3.43)$ & 0.3298 & 1.25 & $(0.48-3.24)$ & 0.8351 & 1.19 & $(0.73-1.95)$ & 0.5567 \\
\hline
\end{tabular}

0 represents the less frequent allele and 1 is the most frequent; $\mathrm{ND}=$ not determined; $\mathrm{OR}=$ odds ratio; $\mathrm{Cl}=$ confidence interval; $\mathrm{HC}=$ healthy control. 


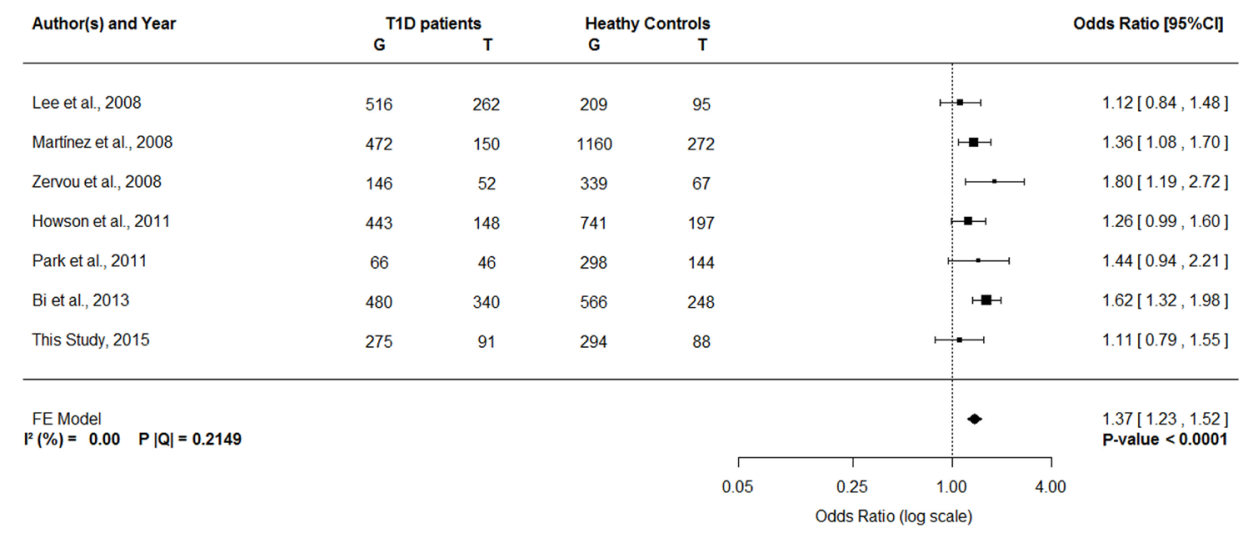

Figure 1. Forest plot from the meta-analysis for the SNP rs7574865 within the STAT4.

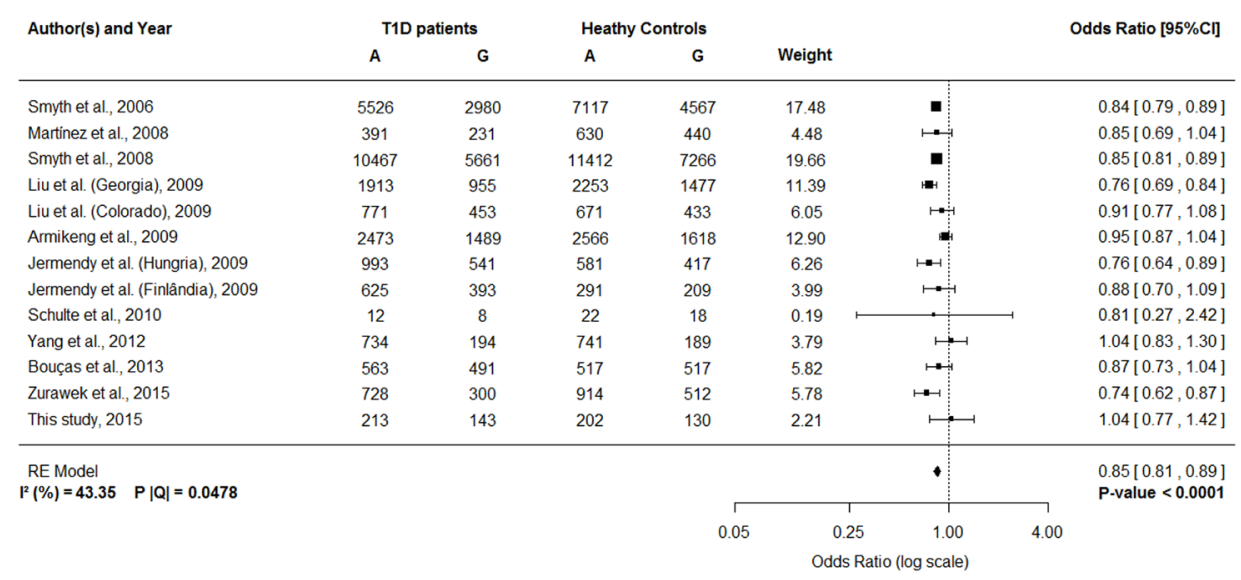

Figure 2. Forest plot from the meta-analysis for the SNP rs7574865 within the IFIH1.

\section{DISCUSSION}

Several T1D patients present simultaneously with autoimmune disorders and organ commitment. APSIII is the most common autoimmune disorder in T1D patients and is found more frequently in adults; it has low incidence in children. The individual pathogenic mechanisms underlying T1D, AITD, and CD remain unknown. Furthermore, it is unclear whether the development of these diseases is due to a shared common etiopathological mechanism or if it is just a consequence of the presence of one autoimmune disorder functioning as a trigger for the insurgence of another. The regulatory pathway of $\mathrm{T}$ cells might be a molecular target to understand the pathogenesis of APSIII due to the involvement of T cell activation in T1D, AITD and CD mechanisms. In addition, the role of viral infection and its related pathways may also be important in understanding the link between T1D, AITD, APSIII, and CD.

In this study, we assessed the role of STAT4 and IFIH1 variants in T1D and APSIII susceptibility. STAT4 is involved in Th1 regulation and its inhibition prevents the development of 
autoimmune diabetes in non-obese diabetic (NOD) mice. Moreover, genetic variants of STAT4 are associated with autoimmune disorders in several populations, making STAT4 an emerging therapeutic target (Yang et al., 2004; Bi et al., 2013; Zheng et al., 2013). IFIH1 SNPs were first associated with T1D in autoimmune disease and have since been associated with other autoimmune systemic disorders. Upon viral infection, IFIH1 activates the IFN-I pathway and the release of IFN-I can activate STAT4, followed by the Th1 gene expression profile in mature dendritic cells (Kariuki et al., 2008). Herein, we investigated the individual and combined influence of STAT4 and IFIH1 variants on T1D and APSIII development. In addition, we examined if STAT4 and IFIH1 SNPS are associated with development of T1D in a meta-analysis study.

The rs7574865 SNP is one of the most frequently examined polymorphisms in the STAT4 gene and its function is related to gene expression on a transcriptional level and splice variation (Liang et al., 2012). The T allele of this particular SNP has been associated with multiple autoimmune disorders but the association is dependent on the population examined (Lee et al., 2010). In the present study, the SNPs examined from STAT4 and IFIH1 did not show any correlation (individually or combined) with incidence of T1D and/or APSIII. Although the rs7574865 SNP is within intron 3, it displays a linkage disequilibrium with other SNPs that have a possible functional consequence (Zheng et al., 2013). The rs3024839 SNP is an intragenic missense mutation ( $>$ > $G$ resulting in isoleucine > valine substitution) with probable functional consequences in STAT4. Although STAT4 SNPs have been frequently studied as potential indicators for autoimmune diseases, the results are still unclear, indicating that STAT4 might play varying roles in these disorders. Our results agree with the meta-analysis performed by Zheng et al. (2013), which revealed that the STAT4 rs7574865 polymorphism is associated with several autoimmune diseases, including SLE, RA, scleroderma, systemic sclerosis (SSc), and primary SS, but is not associated with T1D, ulcerative colitis, and Crohn's disease. Interestingly, SLE, RA, SSc, and SS are considered systemic disorders, whereas T1D is characterized as an organ-specific manifestation, which suggests that mutations in STAT4 are primarily related to systemic rather than organ-specific disorders.

On the other hand, the study performed by Zervou et al. (2008) assessed the link between the rs7574865 SNP and risk of T1D in Crete, where there is a genetically homogenous population, and the results indicated that there was an association. Moreover, this polymorphism was strongly associated with T1D in a northeastern Chinese population (Bi et al., 2013). Additionally, the study performed by Fourati et al. (2012) assessed the possible role of non-HLA genes in APSII, which includes Addison's disease and AITD and/or T1D, in a Tunisian population. Their results indicated that the rs7574865 SNP in STAT4 was associated with APSII but not with T1D or AITD alone, suggesting that STAT4 is involved with the co-occurrence of autoimmune endocrinopathies in APSII individuals.

IFIH1 is a helicase that senses viral dsRNA and, when activated, supports the transcription of IFN-I and IFN-induced genes (Robinson et al., 2011). Since IFIH1 acts during viral infections, we hypothesized in a previous publication that a defective mechanism in virus recognition might be caused by a defective IFIH1 (Moura et al., 2013). However, in the present study, we did not find any association between the $\mathrm{rs} 3747517$ ( $\mathrm{A}>\mathrm{G}$ resulting in histidine $>$ arginine substitution in exon 13) or rs1990760 (C > T resulting in alanine > threonine substitution in exon 15) SNPs in IFIH1 and T1D or APSIII. Despite this, on including our data in the meta-analysis study, the rs 1990760 SNP in IFIH1 was found to be associated with T1D. The overexpression of IFIH1 in murine models is related to a chronic state of IFN-I production (Crampton et al., 2012). In multiple sclerosis (MS), which is an autoimmune disease, IFIH1 and Toll-like receptor 7 (TLR7) are overexpressed. TLR7 
is associated with the IFN-I response and, consequently, the IFN signature (Hundeshagen et al., 2012). The T allele in the rs 1990760 SNP in IFIH1 is associated with increased expression of IFIH1 in peripheral blood mononuclear cells and sensitivity to IFN- $\alpha$ (Rönnblom et al., 2011). Therefore, both STAT4 and IFIH1 exert some control in the IFN-I pathway, and their malfunction leads to an altered immune response.

In conclusion, we did not find any association of SNPS in STAT4 or IFIH1 with T1D development in a northeast Brazilian population. However, the meta-analysis showed an association between the rs7574865 and rs1990760 SNPs in STAT4 and IFIH1, respectively, with T1D, even when our negative associations were included.

\section{Conflicts of interest}

The authors declare no conflict of interest.

\section{ACKNOWLEDGMENTS}

Research supported by the following Brazilian Funding agencies: CAPES (Coordenação de Aperfeiçoamento de Pessoal de Nível Superior), CNPq (Conselho Nacional de Desenvolvimento Científico e Tecnológico) and FACEPE (Fundação de Amparo à Ciência e Tecnologia de Pernambuco).

\section{REFERENCES}

Aminkeng F, Van Autreve JE, Weets I, Quartier E, et al. (2009). IFIH1 gene polymorphisms in type 1 diabetes: genetic association analysis and genotype - phenotype correlation in the Belgian population. Hum. Immunol. 70: 706-710.

Bi C, Li B, Cheng Z, Hu Y, et al. (2013). Association study of STAT4 polymorphisms and type 1 diabetes in Northeastern Chinese Han population. Tissue Antigens 81: 137-140.

Bouças AP, Brondani LA, Souza BM, Lemos NE, et al. (2013). The A allele of the rs1990760 polymorphism in the IFIH1 gene is associated with protection for arterial hypertension in type 1 diabetic patients and with expression of this gene in human mononuclear cells. PLoS One 12: e83451.

Chistiakov DA (2010). Interferon induced with helicase C domain 1 (IFIH1) and virus-induced autoimmunity: a review. Viral Immunol. 23: 3-15.

Crampton SP, Deane JA, Feigenbaum L and Bolland S (2012). Ifih1 gene dose effect reveals MDA5-mediated chronic type I IFN gene signature, viral resistance, and accelerated autoimmunity. J. Immunol. 188: 1451-1459.

Dittmar M and Kahaly GJ (2010). Genetics of the autoimmune polyglandular syndrome type 3 variant. Thyroid 20: 737-743.

Fourati H, Bouzid D, Abida O, Kharrat N, et al. (2012). Non-HLA autoimmunity genetic factors contributing to autoimmune polyglandular syndrome type II in Tunisian patients. Hum. Immunol. 73: 740-746.

Gabir MM, Hanson RL, Dabelea D, Imperatore G, et al. (2000). The 1997 American Diabetes Association and 1999 World Health Organization Criteria for Hyperglycemia. Diabetes Care 8: 1108-1112.

Gillespie KM (2006). Type 1 diabetes: pathogenesis and prevention. Can. Med. Assoc. J. 175: 165-170.

Howson JM, Rosinger S, Smyth DJ, Boehm BO, et al. (2011). Genetic analysis of adult-onset autoimmune diabetes. Diabetes 60: 2645-2653.

Hundeshagen A, Hecker M, Paap BK, Angerstein C, et al. (2012). Elevated type I interferon-like activity in a subset of multiple sclerosis patients: molecular basis and clinical relevance. J. Neuroinflammation 9: 140.

Jaïdane H, Sané F, Gharbi J, Aouni M, et al. (2009). Coxsackievirus B4 and type 1 diabetes pathogenesis: contribution of animal models. Rev. Diabetes Metab. 25: 591-603.

Jermendy A, Szatmári I, Laine AP, Lukács K, et al. (2009). The interferon-induced helicase IFIH1 Ala946Thr polymorphism is associated with type 1 diabetes in both the high-incidence Finnish and the medium-incidence Hungarian populations. Diabetologia 53: 98-102.

Jun HS and Yoon JW (2001). The role of viruses in type I diabetes: two distinct cellular and molecular pathogenic mechanisms 
of virus-induced diabetes in animals. Diabetologia 44: 271-285.

Kariuki SN, Kirou KA, Macdermott EJ, Barillas-Arias L, et al. (2008). Cutting edge: autoimmune Disease risk variant of STAT4 confers increased sensitivity to IFN-alpha in lupus patients in vivo. J. Immunol. 1: 34-38.

Kobayashi S, Ikari K, Kaneko H, Kochi Y, et al. (2008). Association of STAT4 with susceptibility to rheumatoid arthritis and systemic lupus erythematosus in the Japanese population. Arthritis Rheum. 58: 1940-1946.

Kordonouri O, Klinghammer A, Lang EB, Gruters-Kieslich A, et al. (2002). Thyroid autoimmunity in children and adolescents with type 1 diabetes: a multicenter survey. Diabetes Care 25: 1346-1350.

Lee HS, Park H, Yang S, Kim D, et al. (2008). STAT4 polymorphism is associated with early-onset type 1 diabetes, but not with late-onset type 1 diabetes. Ann. N.Y. Acad. Sci. 1150: 93-98.

Lee YH, Woo JH, Choi SJ, Ji JD, et al. (2010). Association between the rs7574865 polymorphism of STAT4 and rheumatoid arthritis: a meta-analysis. Rheumatol. Int. 30: 661-666.

Levy DE and Darnell JE Jr (2002). Stats: transcriptional control and biological impact. Nat. Rev. Mol. Cell Biol. 3: 651-662.

Liang YL, Wu H, Shen X, Li PQ, et al. (2012). Association of STAT4 rs7574865 polymorphism with autoimmune diseases: a meta-analysis. Mol. Biol. Rep. 39: 8873-8882.

Liu S, Wang H, Jin Y, Podolsky R, et al. (2009). IFIH1 polymorphisms are significantly associated with type 1 diabetes and IFIH1 gene expression in peripheral blood mononuclear cells. Hum. Mol. Genet. 18: 358-365.

Martínez A, Varadé J, Márquez A, Cénit MC, et al. (2008a). Association of the STAT4 gene with increased susceptibility for some immune-mediated diseases. Arthritis Rheum. 58: 2598-2602.

Martínez A, Santiago JL, Cénit MC, de Las Heras V, et al. (2008b). IFIH1-GCA-KCNH7 locus: influence on multiple sclerosis risk. Eur. J. Hum. Genet. 16: 861-864.

Moura R, Araujo J, Guimarães R, Crovella S, et al. (2013). Interferon induced with helicase C domain 1 (IFIH1): Trends on helicase domain and type 1 diabetes onset. Gene 516: 66-68.

Palomino-Morales RJ, Diaz-Gallo LM, Witte T and Anaya JM (2010). Influence of STAT4 polymorphism in primary Sjögren's syndrome. J. Rheumatol. 37: 1016-1019.

Park Y, Lee HS, Park Y, Min D, et al. (2011). Evidence for the role of STAT4 as a general autoimmunity locus in the Korean population. Diabetes Metab. Res. Rev. 27: 867-871.

Robinson T, Kariuki SN, Franek BS, Kumabe M, et al. (2011). Autoimmune disease risk variant of IFIH1 is associated with increased sensitivity to IFN- $\alpha$ and serologic autoimmunity in lupus patients. J. Immunol. 187: 1298-1303.

Rönnblom L, Alm GV and Eloranta ML (2011). The type I interferon system in the development of lupus. Semin. Immunol. 23: 113-121.

Salminen K, Sadeharju K, Lönnrot M, Vähäsalo P, et al. (2003). Enterovirus infections are associated with the induction of beta-cell autoimmunity in a prospective birth cohort study. J. Med. Virol. 69: 91-98.

Schulte BM, Bakkers J, Lanke KHW, Melchers WJG, et al. (2010). Detection of enterovirus RNA in peripheral blood mononuclear cells of type 1 diabetic patients beyond the stage of acute infection. Viral Immunol. 23: 99-104.

Smyth DJ, Cooper JD, Bailey R, Field S, et al. (2006). A genome-wide association study of nonsynonymous SNPs identifies a type 1 diabetes locus in the interferon-induced helicase (IFIH1) region. Nat. Genet. 38: 617-619.

Smyth DJ, Plagnol V, Walker NM, Cooper JD, et al. (2008). Shared and distinct genetic variants in type 1 diabetes and celiac disease. N. Engl. J. Med. 359: 2767-2777.

Stark K, Rovenský J, Blazicková S, Grosse-Wilde H, et al. (2009). Association of common polymorphisms in known susceptibility genes with rheumatoid arthritis in a Slovak population using osteoarthritis patients as controls. Arthritis Res. Ther. 11: R70.

Viechtbauer W (2010). Conducting meta-analyses in R with the metafor package. J. Stat. Soft. 36: 1-48.

Yang Z, Chen M, Ellett JD, Fialkow LB, et al. (2004). Autoimmune diabetes is blocked in Stat4-deficient mice. J. Autoimmun. 22: $191-200$.

Yang HUI, Wang Z, Xu K, Gu R, et al. (2012). IFIH1 gene polymorphisms in type 1 diabetes: genetic association analysis and genotype-phenotype correlation in Chinese Han population. Autoimmunity 3: 226-232.

Zervou MI, Mamoulakis D, Panierakis C, Boumpas DT, et al. (2008). STAT4: a risk factor for type 1 diabetes? Hum. Immunol. 69: 647-650.

Zheng J, Yin J, Huang R, Petersen F, et al. (2013). Meta-analysis reveals an association of STAT4 polymorphisms with systemic autoimmune disorders and anti-dsDNA antibody. Hum. Immunol. 8: 986-992.

Zurawek M, Fichna M, Fichna P, Skowronska B, et al. (2015). Cumulative effect of IFIH1 variants and increased gene expression associated with type I diabetes. Diabetes Res. Clin. Pract. 107: 259-266. 